\title{
Las entidades asociativas prioritarias como instrumento de integración de empresas agroalimentarias. Dificultades y oportunidades de su reconocimiento
}

\author{
Daniel Hernández Cáceres ${ }^{1}$
}

Recibido: 30 de octubre de 2017 / Aceptado: 14 de febrero de 2018

Resumen. Ante la atomización existente en el sistema cooperativo español y la consiguiente falta de nivel competitivo de las cooperativas españolas en el mercado internacional, se desarrolló la Ley 13/2013, de 2 de agosto, de fomento de la integración de cooperativas y de otras entidades asociativas de carácter agroalimentario, que pretendía hacer frente a estos problemas. Obteniendo la calificación de "Entidad Asociativa Prioritaria" creada por dicha Ley, dichas entidades tendrán acceso preferencial a subvenciones y ayudas, lo mismo que las entidades que se encuentren integradas en ellas y los productores agrarios. Pero para la obtención de esta calificación se van a encontrar con múltiples dificultades, ya sean intrínsecas de la propia integración como pueden ser los problemas de la concentración/diferenciación, o los requisitos impuestos por la misma Ley y el Real Decreto $550 / 2014$, de 27 de junio, por el que se desarrollan los requisitos y el procedimiento para el reconocimiento de las Entidades Asociativas Prioritarias y para su inscripción y baja en el Registro Nacional de Entidades Asociativas Prioritarias, entre los que se encuentran las exigencias de facturación, comercialización conjunta y el controvertido carácter de supra-autonomía, o con dificultades derivadas de la pluralidad normativa generada por las comunidades autónomas.

Palabras clave: Cooperativas; Grupos Cooperativos; Cooperativas de Segundo Grado; Redimensionamiento; Supraautonomía.

Claves Econlit: K29; P13; Q13.

\section{[en] Associative priority entities as an instrument for the integration of agrifood companies. Difficulties and opportunities of its recognition}

\begin{abstract}
Given the existing fragmentation in the Spanish cooperative system and the consequent lack of competitive level of Spanish cooperatives in the international market, the Act 13/2013, of August $2 \mathrm{nd}$, to promote the integration of cooperatives and other associative agri-food entities was developed, which sought to address these problems. Obtaining the qualification of "Associative Priority Entity" created by said Act, these entities will have preferential access to subsidies and aids, as well as the entities that are integrated in them and the agrarian producers. But to obtain this qualification they will find multiple difficulties, whether they are intrinsic to the integration itself, such as the problems of concentration / differentiation, or the requirements imposed by the same Act and by the Royal Decree 550/2014, of June 27th, by which the requirements and the procedure for the recognition of the Associative Priority Entities and for their inscription and entry in the National Register of Associative Priority Entities are developed, among which are the demands for turnover,
\end{abstract}

1 Universidad de Almería, España

Dirección de correo electrónico: danielhernandezcaceres@gmail.com 
joint marketing and the controversial nature of supra-regional, or difficulties derived from the plurality of regulations generated by the autonomous regions.

Keywords: Cooperatives; Cooperative Groups; Second Tier Cooperatives; Resizing; Supra-regional autonomy.

Sumario. 1. Introducción. 2. El fomento de la integración. 3. Oportunidades del reconocimiento como Entidad Asociativa Prioritaria. 4. Dificultades para la obtención del reconocimiento de Entidad Asociativa Prioritaria. 5. Iniciativas desarrolladas por las Comunidades Autónomas. 6. Conclusiones. 7. Referencias bibliográficas.

Cómo citar: Hernández Cáceres, D. (2017) Las entidades asociativas prioritarias como instrumento de integración de empresas agroalimentarias. Dificultades y oportunidades de su reconocimiento. REVESCO. Revista de Estudios Cooperativos, Monográfico, № 126, pp. 198-211. DOI: 10.5209/REVE.59766.

\section{Introducción}

La entrada en vigor de la Ley 13/2013, de 2 de agosto, de fomento de la integración de cooperativas y de otras entidades asociativas de carácter agroalimentario (en adelante LFIC), desarrollada reglamentariamente por medio del Real Decreto $550 / 2014$, de 27 de junio, por el que se desarrollan los requisitos y el procedimiento para el reconocimiento de las Entidades Asociativas Prioritarias y para su inscripción y baja en el Registro Nacional de Entidades Asociativas Prioritarias, previsto en la Ley 13/2013, de 2 de agosto, de fomento de la integración de cooperativas y de otras entidades asociativas de carácter agroalimentario (en adelante REAP) ha supuesto una de las últimas medidas para tratar de impulsar y fomentar la integración cooperativa y asociativa con la intención de favorecer la competitividad, el redimensionamiento, la modernización y la internacionalización de dichas entidades, tal y como se indica en su exposición de motivos. En el año 2013 la cadena agroalimentaria española presentaba una clara asimetría ${ }^{2}$. Nos encontrábamos ante un sector claramente marcado por la atomización, con un gran número de cooperativas de una reducida dimensión en comparación con la situación del resto de países de la $\mathrm{UE}^{3}$. Esta desintegración va a provocar que las pequeñas cooperativas españolas se encuentren con dificultades

2 Así lo entendió el ejecutivo al establecer en el Informe sobre el anteproyecto de Ley de fomento de la integración cooperativa que "la cadena agroalimentaria española presentaba una asimetría que debía ser corregida mediante la puesta en marcha, entre otras actuaciones, de políticas e instrumentos legislativos que mejoren la estructura comercializadora".

3 Según datos del Plan Estatal de Integración Asociativa 2015-2020: El sistema agroalimentario español estaba compuesto por 3.844 Entidades Cooperativas, con una facturación aproximada de 25.000 millones de euros, más de un millón de socios y cerca de 100.000 empleos directos, representando un sector básico en el entramado productivo, industrial y comercializador.

La atomización y la falta de competitividad quedan demostradas cuando comparamos el sector agroalimentario español con el del resto de países europeos y es que ninguna cooperativa española está entre las 25 más grandes de la Unión Europea. La mayor cooperativa europea facturó más de 11.000 millones de euros en 2013 y la mayor de España apenas superó los 900 millones. La facturación de todas las cooperativas españolas es similar a la de veintiocho cooperativas de Dinamarca. 2.400 cooperativas francesas facturan más de tres veces la cifra de las españolas. La facturación media en el conjunto del cooperativismo agroalimentario en España, es de 6,7 millones de euros, en contraposición con la media de 380 millones de euros de las 10 más grandes de la Unión Europea. 
para alcanzar deseables niveles de competitividad frente a las grandes compañías internacionales, pudiendo verse comprometidos en un futuro los productos comercializados por las entidades españolas (Berges, 2014). Y es que el requisito dimensional a la hora de competir en los mercados internacionales es crítico, ya que llevar a cabo una política exportadora en la que se movilicen grandes cantidades de recursos va a ser un problema para las organizaciones de menor tamaño. Por lo que o las cooperativas crecen o están abocadas a la inexistencia en el mercado internacional ${ }^{4}$.

\section{El fomento de la integración}

Ante esta situación de atomización resultaba necesario poner en marcha medidas que fomentaran la integración y la potenciación de grupos comercializadores de base cooperativa y asociativa, que posibilitaran a estas entidades operar en toda la cadena agroalimentaria, tanto en los mercados nacionales como en los internacionales y creando un nuevo modelo que repercuta favorablemente en los productores agrarios situados en los eslabones inferiores de la cadena, propiciando el aumento de las rentas obtenidas. A la vez se pretende establecer un sistema racional y eficiente de infraestructuras agroalimentarias en las zonas rurales, que permita una inversión en tecnologías e investigación, que aumente la productividad y la competitividad de estas entidades en el mercado, y que contribuya a la valorización y comercialización de sus producciones. De esta manera se cumple con el mandato del artículo 129.2 de la Constitución española (CE), fomentando las sociedades cooperativas e impulsando un modelo cooperativo competitivo que se adapte a las exigencias del mercado global.

La LFIC se presenta como una de las principales medidas para tratar de reducir esta atomización, favoreciendo la integración de cooperativas y de otras entidades asociativas agroalimentarias de ámbito supraautonómico, mediante la creación de una nueva categoría denominada "Entidad Asociativa Prioritaria" (en adelante EAP). Las entidades asociativas agroalimentarias a las que se les reserva expresamente la posibilidad de obtener el reconocimiento como EAP son: 1) Cooperativas y sociedades cooperativas agroalimentarias, 2) Grupos cooperativos, 3) Sociedades agrarias de transformación, 4) Organizaciones de productores con personalidad jurídica propia, 5) Entidades civiles o mercantiles, siempre que más del 50 por ciento de su capital pertenezca a sociedades cooperativas, a organizaciones de productores o a sociedades agrarias de transformación. En el caso de que estas entidades económicas tengan la forma de SA, sus acciones deberán ser nominativas (art. 1.3 LFIC y art. 2.1 REAP). Las entidades de este tipo, existentes o que se constituyan tras un proceso de integración de varias de ellas, que cumplan con los requisitos establecidos en la Ley y el Reglamento, podrán obtener dicho reconocimiento tras solicitarlo.

4 Este tamaño también facilitará las inversiones requeridas en productos y procesos, la creación de sociedades con otros agentes, el acceso a tecnologías de información y comunicación; y el acceso a la financiación (Fayos y Calderón, 2013). 
A pesar de que a la hora de seleccionar los tipos la LFIC hace referencia a que son entidades que, además de vertebrar la producción agraria, contribuyen y mejoran la cohesión territorial generando empleo y ofreciendo servicios que demandan los ciudadanos del medio rural ${ }^{5}$, puede hablarse también de un fomento de entidades de economía social (Cano, 2015). La mayoría de los tipos incluidos son entidades con una marcada tendencia hacia la economía social, en la que la repartición de beneficios no está directamente relacionada con el capital social aportado por cada socio, ni tampoco la toma de decisiones. Cuando se habla de cooperativas y de grupos de cooperativas la pertenencia a la economía social está expresamente recogida en el artículo 5 de la Ley 5/2011 de Economía Social. Con respecto a las Sociedades Agrarias de Transformación apenas existe ya duda de que representan "una figura jurídica intermedia próxima a las sociedades cooperativas y que tradicionalmente se ha incluido por su naturaleza mutualista entre las empresas de economía social." (Vargas, 2012: 35). Las Organizaciones de Productores únicamente pertenecerán a esta economía social cuando se trate de una sección de una cooperativa creada en el marco de la normativa reguladora de este tipo de entidades, o bien un grupo de productores de una SAT que constituya una sección similar que cumpla con los requisitos establecidos en la normativa que regula las secciones de las cooperativas y respete la suya propia ${ }^{6}$.

Lo que se pretende con el reconocimiento como EAP en esencia es fomentar la integración de cooperativas y otras entidades asociativas, consiguiendo que al aumentar de tamaño se aumente la competitividad y la rentabilidad del sector (Calvo, 2013). Este fomento de la integración se encuentra de forma directa cuando entre las entidades que pueden ser reconocidas incluye a los grupos cooperativos, formados por varias cooperativas con una entidad cabeza de grupo que ejercita facultades o emite instrucciones de obligado cumplimiento para las cooperativas agrupadas, de forma que se produzca una unidad de decisión en el ámbito de dichas facultades (art. 78.1 Ley 27/1999, de 16 de julio, de Cooperativas, en adelante LCOOP), sin que en ningún caso este reconocimiento a los grupos cooperativos provoque la exclusión de la utilización de diferentes procesos de integración. La propia LFIC en la búsqueda del fomento de la integración también recoge la posibilidad de acudir a mecanismos donde la integración de la cooperativa es total, como son el caso de la fusión por constitución de nueva entidad, conllevando la desaparición de las entidades originales que pasarán a integrarse en esa nueva entidad, o el caso de la fusión por absorción en la que una de las entidades originales desaparece al ser absorbida por la otra, o también es posible que se acuda a un mecanismo mucho más flexible como es la constitución de una cooperativa de segundo grado, de modo que continúen existiendo las entidades originales de base tras la creación de esta nueva entidad de grado superior que asuma las tareas de comercialización en común de todos los socios pertenecientes a dichas entidades. Corresponderá a cada una de las entidades elegir con qué otras entidades van a integrarse y qué mecanismo se pondrá en marcha para ello.

\footnotetext{
Exposición de Motivos LFIC.

6 Art 154 Reglamento (UE) N. ${ }^{\circ}$ 1308/2013 del Parlamento Europeo y del Consejo, de 17 de diciembre de 2013, por el que se crea la organización común de mercados de los productos agrarios (en adelante ROCMPA): "la organización de productores que lo haya solicitado deberá ser una entidad jurídica o ser parte claramente definida de una entidad jurídica".
} 


\section{Oportunidades del reconocimiento como Entidad Asociativa Prioritaria}

Gracias al aumento de tamaño conseguido debido a la integración, las entidades agroalimentarias surgidas van a obtener una mayor capacidad competitiva en el mercado, y consecuentemente los productores agrarios pertenecientes a estas entidades también verán como sus rentas aumentan. Pero además los poderes públicos han querido incentivar la integración de las diferentes entidades mediante el acceso a otros beneficios, respetando en todo caso la normativa europea en cuanto ayudas estatales. De esta manera la LFIC, fomentando la integración, regula otra serie de oportunidades para las entidades que obtengan el reconocimiento como EAP. La principal consecuencia es el acceso preferente a determinadas ayudas y subvenciones del Ministerio de Agricultura y Pesca, Alimentación y Medio Ambiente y de las Comunidades Autónomas, que podrán ser disfrutadas tanto por la entidad asociativa calificada como prioritaria, como por sus entidades asociadas y los productores primarios en ellas asociados.

Concretamente las EAPs tendrán preferencia en: 1) subvenciones y ayudas en materia de inversiones materiales o inmateriales destinadas a mejorar los procedimientos de gestión y comercialización, los procedimientos de gestión y transformación y su competitividad y orientar su producción al mercado; 2) el acceso a actividades formativas y de cooperación; 3) en materia de internacionalización, de promoción y de $\mathrm{I}+\mathrm{D}+\mathrm{i}$; 4) el acceso a las líneas ICO de financiación preferente; 5) las actuaciones contempladas en los Programas de Desarrollo Rural a favor de la competitividad, la transformación y la comercialización (art. 4.1 LFIC).

Las entidades asociativas integradas en EAPs gozarán de preferencia en: 1) subvenciones y ayudas en materia de inversiones materiales o inmateriales destinadas a mejorar los procedimientos de gestión y comercialización, los procedimientos de gestión y transformación y su competitividad y orientar su producción al mercado; 2) actividades formativas y de asistencia técnica; contrataciones de pólizas que puedan establecerse para estas entidades en el marco del Sistema de Seguros Agrarios Combinados; 3) acceso a programas o actuaciones en I+D+i y en nuevas tecnologías; 4) acceso a programas o actuaciones enfocadas específicamente a la mejora de la competitividad, la transformación y la comercialización (art. 4.2 LFIC).

Mientras que los productores agrarios que formen parte de EAPs o de las Entidades Asociativas integradas tendrán prioridad en subvenciones y ayudas para mejorar su competitividad y orientar su producción al mercado en el marco de la EAP de la que formen parte, respecto a los productos para los que ha sido reconocida (art. 4.3 LFIC).

Estas ayudas y subvenciones se concretan en un conjunto de medidas para el que se ha presupuestado una contribución pública de más de 256 millones de euros en el periodo 2014-20207. El Ministerio de Agricultura y Pesca, Alimentación y

Tal y como se desprende de la Resolución de 20 de febrero de 2017, del Fondo Español de Garantía Agraria, por la que se convocan ayudas a inversiones materiales o inmateriales en transformación, comercialización y desarrollo de productos agrarios, en el marco del Programa Nacional de Desarrollo Rural 2014-2020, el presupuesto total previsto en la submedida de inversiones, en la convocatoria de los años 2017-18 es de un total de 34.912.779,27 euros, distribuidos en dos anualidades: 18.195.146,87 euros en 2017 y $16.717 .632,40$ 
Medio Ambiente ha apuntado que en principio el reconocimiento obtenido como EAP no excluye de otras ayudas, pudiendo solicitarse ante distintas administraciones, siempre y cuando no se destinen a la misma finalidad y objeto. Por tanto, no se podrá solicitar una subvención para construir la misma instalación a través de dos líneas de ayuda distintas.

\section{Dificultades para la obtención del reconocimiento de Entidad Asociativa Prioritaria}

Las entidades susceptibles de obtener la calificación como EAP se van a encontrar varias dificultades reflejadas en su mayoría en los requisitos que vienen impuestos por la LFIC y el REAP. Estos condicionantes en ocasiones resultarán casi inalcanzables para las entidades, debido unas veces a la excesiva cuantía exigida, otras veces a la exigencia de la supra-autonomía y el papel decisivo de las Comunidades Autónomas en este ámbito, o la obligatoriedad de conseguir una comercialización conjunta de la totalidad de la producción.

\subsection{El difícil equilibrio entre concentración y diferenciación}

Antes de iniciar con las exigencias de la LFIC, habría que destacar en primer lugar la dificultad que aparece siempre que se habla de integración entre entidades, la relacionada con el tamaño ideal de la cooperativa (concentración/diferenciación). Como se ha comentado anteriormente, la integración se podrá llevar a cabo a través de los mecanismos antes descritos, los cuales tendrán unos efectos distintos para las entidades originales. Los procedimientos de fusión supondrán una gran concentración en la entidad resultante, pero a la vez se perderá la identidad de las entidades originales. Por el contrario, acudir a los grupos cooperativos y a las cooperativas de segundo grado proporcionará una mayor flexibilidad, pero disminuirá esa concentración. Los socios históricamente se han mostrado reticentes ante la posible pérdida de autonomía que supondría la integración, o por la importancia que tiene para ellos la identidad, la diferenciación existente, la cual se perdería si se produjese el cambio de la denominación social. Así pues, habrá que analizar cuál de estos procedimientos es el más conveniente para el caso concreto y dotar de mecanismos jurídicos adecuados a dicha entidad para tratar de evitar la disolución de la nueva entidad surgida (Vargas, 2010).

Tampoco se puede obviar que estas integraciones de cooperativas agroalimentarias van a desencadenar un movimiento dentro de los puestos de dirección y gestión de dichas entidades, provocando entre los miembros un temor a

\footnotetext{
euros en 2018. La financiación de estas ayudas se realizará en un 53 por ciento con cargo al FEADER y en un 47 por ciento con cargo al presupuesto nacional. Además, se establecen unos márgenes a dichas ayudas, que podrán alcanzar hasta un 40 por cien de los gastos subvencionables, con un límite de 5.000.000 euros por proyecto de inversión.

Por su parte, las convocatorias de ayudas para acciones de formación y para actividades de demostración e información relacionadas con la actividad de las EAP superarán los 400.000 euros para cada una de ellas en la anualidad 2017. Y con respecto a las submedidas de cooperación para proyectos de suministro sostenible de biomasa y prácticas medioambientales en curso, estas superaran los 200.000 euros en 2017 en su conjunto.
} 
la pérdida de los puestos que ocupan. Este será otro de los problemas derivados de la integración. No hay que olvidar el marcado individualismo que caracteriza a los empresarios españoles, reflejado en el tejido empresarial dominado de manera absoluta por PYMEs y microempresas (Vargas, 2010). En todo caso será preciso que a la cabeza de estas nuevas agrupaciones, en los puestos de los responsables de la dirección y gestión, se sitúen personas profesionales formadas y con experiencia en las nuevas herramientas e instrumentos de gestión.

\subsection{El requisito de facturación}

Dentro de los requisitos exigidos para poder ser reconocida como EAP encontramos la facturación de la entidad asociativa solicitante, o la suma de las facturaciones de las entidades que se fusionan o integran, referentes a cualquiera de los tres últimos ejercicios económicos cerrados, previos a la solicitud (art. 3.1.d LFIC, art. 2, apartados 2, 3 y 6 REAP). Ahora bien, las cantidades varían en función del producto para el que se solicite el reconocimiento, por lo que encontramos las siguientes posibilidades: 1) Si se solicita el reconocimiento para un producto determinado, su facturación anual, correspondiente a la producción comercializada de dicho producto, deberá ser superior a la cuantía que se señala en el apartado a) del anexo I del REAP. En él encontramos cantidades que comprenden desde los 20 millones de euros como en el caso del algodón o de la producción forestal, hasta los 650 millones como en el caso de la leche y los productos lácteos; 2) Si se solicita un reconocimiento genérico, su facturación total anual correspondiente al conjunto de productos comercializados por la entidad, deberá ser superior a la cuantía que se señala en el apartado b) del anexo I, en concreto 750 millones de euros. Sin embargo, en ambos reconocimientos las cantidades se verán reducidas en un $30 \%$ cuando se traten de sociedades cooperativas de primer grado.

Las altas cuotas exigidas hacen que únicamente muy pocas entidades, integrándose entre sí, puedan obtener dicho reconocimiento. Según los datos de OSCAE de 2015, del TOP 10 de Cooperativas españolas, únicamente alcanzarían dichos requisitos de facturación: COREN $\left(1^{\mathrm{a}}\right)$, DCOOP $\left(2^{\mathrm{a}}\right)$, SAT LECHERA ASTURIANA $\left(4^{\mathrm{a}}\right)$ y AGROSEVILLA ACEITUNAS $\left(9^{\mathrm{a}}\right)$.

\subsection{Carácter suprautonómico}

Otra de las condiciones que tienen un marcado peso a la hora de solicitar el reconocimiento es la obligatoriedad de que la entidad cuente con una implantación y un ámbito de actuación económico de carácter supra-autonómico (art. 3.1.b LFIC). A los efectos de la determinación de esta supra-autonomía se establece que se entenderá que existe cuando simultáneamente dispone de socios en más de una comunidad autónoma, sin que exceda del $90 \%$ en el ámbito de una concreta - en el caso de sociedades de capital solo se tendrán en cuenta las entidades integradas en ellas- y su actividad económica no exceda del $90 \%$ en una Comunidad Autónoma concreta (art. 2.5 REAP).

Ahora bien, la norma readapta estos porcentajes para hacer frente tanto a la realidad de la producción estatal, con la existencia de producciones que se 
concentran en una sola Comunidad Autónoma, como a las diferentes composiciones de las Comunidades Autónomas. Así pues, los porcentajes sociales y económicos pasarán a ser del $95 \%$ cuando la producción nacional del producto determinado se localice en más de un $60 \%$ en una única Comunidad Autónoma o cuando la entidad desarrolle su actividad en más de un $50 \%$ en una Comunidad Autónoma con cinco o más provincias.

Se podría afirmar que dicho carácter supra-autonómico ha sido introducido con la intención de que no pudiera sufrir las consecuencias de un recurso de inconstitucionalidad por parte de alguna Comunidad Autónoma ${ }^{8}$ que podía ver afectadas sus competencias en materia de cooperativas (Vargas, 2013). Hay que recordar que la competencia sobre el régimen de cooperativas recae en las Comunidades Autónomas ${ }^{9}$ que la hayan asumido en su Estatuto. Por lo que en nuestro ordenamiento jurídico prodigan las normas autonómicas, una por cada Comunidad Autónoma excepto Canarias, junto con una Ley estatal ${ }^{10}$ que únicamente será de aplicación para las Cooperativas que desarrollen su actividad cooperativizada en el territorio de varias Comunidades Autónomas, excepto cuando en una de ellas se desarrolle con carácter principal, y en aquellas que realicen principalmente su actividad cooperativizada en las ciudades de Ceuta y Melilla. Esta pluralidad legislativa provocará complicaciones en los procesos de integración supra-autonómicos, como es en el caso de las fusiones, ya que no existe una solución a los conflictos que surjan entre las normas autonómicas aplicables (Cano, $2015)^{11}$.

\subsection{Comercialización conjunta de la totalidad de la producción}

Para obtener el reconocimiento se exige que conste expresamente en los estatutos o en las disposiciones reguladoras de la entidad, la obligación de los productores de entregar la totalidad de su producción, para su comercialización en común, sin perjuicio de las excepciones o limitaciones que por disposición legal o normativa comunitaria sean de aplicación. (art. 3.1.e LFIC y art. 3.2.c. $1^{\mathrm{o}}$ REAP). Esta obligación de aportar el $100 \%$ de la producción ya venía recogida para las

8 Con respecto a los artículos referentes al reconocimiento de la entidad y los cambios que pudieran afectar a su condición de prioritaria, la Generalitat de Cataluña presentó un recurso de inconstitucionalidad por vulneración de las competencias de Cataluña en materia de agricultura con la consiguiente ST 85/2015 de 30 abril del TC, en los que se introdujo el requisito de realizar una consulta previa a las Comunidades Autónomas afectadas por su carácter supra-autonómico.

9 En la Constitución Española (CE) no se hace alusión a las cooperativas en el artículo 149.1 referente a las competencias exclusivas del Estado y así lo decretó la STC 72/1983: "En concreto, la Constitución no reserva de modo directo y expreso competencia alguna al Estado en materia de cooperativas y, en consecuencia, de acuerdo con el art. 149.3 de la propia Norma, la Comunidad tiene las competencias que haya asumido en su Estatuto, correspondiendo al Estado las no asumidas".

10 Ley 27/1999, de 16 de julio, de Cooperativas (LCOOP).

11 Tal y como expone Cano, 2015: 72: "Aunque la doctrina se ha ocupado en ocasiones de esta problemática, pidiendo de forma generalizada una Ley armonizadora, no se ha visto satisfecha esta petición. Por todo ello, la doctrina considera complicada la fusión supra-autonómica de cooperativas, y en muchas ocasiones se huye hacia otras alternativas de integración como las cooperativas de segundo grado, la creación de grupos cooperativos o desarrollar acuerdos intercooperativos". 
organizaciones de productores de frutas y hortalizas ${ }^{12}$, por lo que para este tipo de entidad no va a presentar mayores problemas.

En el ámbito de las cooperativas, esta obligatoriedad que tiene el socio de entregar toda su producción puede identificarse con el "principio de exclusividad", sistema que "se ajusta a la estructura "ortodoxa" de lo que debe ser una cooperativa: es decir, una entidad constituida para realizar actividades empresariales, encaminadas a satisfacer las necesidades y aspiraciones económicas y sociales de sus socios (art. 1 LC)" (Gallego, 2011: 70). Ahora bien, a nivel estatal no existe una obligación general por parte de los socios de aportar la totalidad de su producción, sino que éstos deberán participar en las actividades cooperativizadas en la "cuantía mínima" que establezcan sus Estatutos (art. 15.2b LCOOP), como tampoco existe en otras normas de ámbito autonómico. Esto provoca que nos encontremos ante numerosas situaciones en las que los propios estatutos de las cooperativas imponen únicamente unos porcentajes mínimos de aportación, generando que en ocasiones los propios socios de la cooperativa se conviertan en competidores directos de la cooperativa una vez que el producto pasa a suministrarse. Este cambio en los porcentajes de aportación supondrá un problema en aquellas cooperativas en las que no rige el principio de exclusividad. En caso de incumplimiento por parte de algún socio, la entidad perdería la calificación, sin que se derive ninguna otra consecuencia legal para el socio, que sí que podrá verse afectado por las sanciones contempladas en los estatutos por incumplimiento en la participación.

Por último, señalar que esta exigencia de recoger en los estatutos esta obligación, va a alcanzar no solo a los estatutos que regulen la EAP, sino también a los estatutos correspondientes a las distintas entidades que la componen (art. 3.1.e LFIC).

\subsection{Control democrático}

Se exigen previsiones que garanticen el control democrático de su funcionamiento y de sus decisiones, así como para evitar la posición de dominio de uno o varios de sus socios, que deberán contenerse en los estatutos de la EAP y de las entidades que la integran (art. 3.1.f LFIC y art. 3.2.c. $2^{\circ}$ REAP). Dependiendo de qué tipo de entidad solicite este reconocimiento, el control democrático se realizará de diferente manera, pero ante todo este control democrático ha de garantizar la ausencia de posición de dominio de uno o varios de los socios. Así pues, cuando se trate de una Cooperativa agroalimentaria, todos los socios tendrán derecho a la asistencia a la Asamblea General en la que cada socio tendrá un voto, no obstante, se podrá establecer en los Estatutos la posibilidad de un voto plural ponderado, en función del volumen de la actividad cooperativizada del socio, sin que pueda ser superior en ningún caso a cinco votos sociales y sin que un solo socio pueda poseer más de un tercio de los votos totales de las cooperativas (art. 26.4LC ${ }^{13}$ ), por lo que en el caso de las cooperativas se consigue evitar la posición de dominio. Además se

12 Artículo 5.2 Real Decreto 532/2017 por el que se regulan el reconocimiento y el funcionamiento de las organizaciones de productores del sector de frutas y hortalizas.

13 Para determinar el control democrático de cada una de las Cooperativas que integren esta EAP, habrá que acudir a la norma autonómica que le corresponda. 
les impone a las Cooperativas agroalimentarias la obligación de respetar el principio de libre adhesión voluntaria y abierta (3.2.c. ${ }^{\circ}$ REAP). Cuando la entidad que solicite el reconocimiento sea una SAT, como ocurre con las cooperativas, cada socio dispondrá de un voto, salvo para la adopción de acuerdos que entrañen obligaciones económicas para los socios donde los Estatutos podrán establecer un sistema de voto en proporción a la participación en el capital social (art. 11.2 Real Decreto 1776/1981 por el que se aprueba el Estatuto que regula las Sociedades Agrarias de Transformación en adelante ESAT). Aun así, tampoco nos encontraremos una posición de dominio ya que las aportaciones de los socios no podrán exceder de un tercio del capital social (art. 8.5 ESAT). En el caso de que la entidad solicitante sea una organización de productores el control democrático es uno de los requisitos exigidos para que se constituyan como tales (art. 153.2.c ROCMPA). Finalmente, para el caso de que se trate de una entidad mercantil, cada socio ostentará un derecho de voto en función de su participación en el capital social (art. 188 Ley de Sociedades de Capital), por lo que un socio no podrá tener más del $49 \%$ del capital social de la entidad mercantil que solicite el reconocimiento. Además, será preciso que más del 50 por ciento de su capital pertenezca a varias Sociedades Cooperativas, a Organizaciones de Productores o a Sociedades Agrarias de Transformación (art. 1.3 LFIC y art. 2.1REAP), no pudiendo concentrar una sola de ellas más de ese $49 \%$ para evitar la posición de dominio.

El principal dilema que plantea este requisito es el de delimitar hasta qué eslabón de la cadena asociativa alcanza esta prohibición de posición de dominio, ya que se prohíbe tanto en la EAP como en las sociedades que la integran. Cabe recordar que en las Organizaciones de Productores el requisito de la inexistencia de una posición de dominio se extiende por todos los eslabones de la cadena hasta llegar al eslabón más inferior. No creo que en este caso se exija hasta esos límites tan exagerados, sino que seguramente se limite únicamente a la dirección y gestión de la entidad resultante que tenga reconocida la calificación de EAP, así como la de los eslabones inmediatamente inferiores a la misma.

\section{Iniciativas desarrolladas por las Comunidades Autónomas}

Para tratar de favorecer la integración se podrá trabajar a nivel estatal o a nivel autonómico ya que las ayudas a dichas entidades podrán ser financiadas por la Administración General del Estado y por las de las Comunidades Autónomas (art. 6 LFIC). Las medidas que incorporen ambas normativas deberán ir orientadas en la misma dirección, es decir, las EAP regionales se han de situar en un paso previo al de las EAP estatales, favoreciendo la dimensión de dichas entidades mediante procesos de integración, pero siempre respetando la misma línea marcada por esta normativa estatal, para que estas EAP regionales puedan en el futuro ganar una mayor dimensión, integrarse con entidades de otra comunidad y constituirse como estatales, y que no se encuentren con grandes dificultades al requerírsele unas exigencias totalmente antagónicas a las exigidas por su Comunidad Autónoma. Hasta el momento ya son tres las Comunidades que han regulado las EAP 
regionales, estableciendo medidas para favorecerlas e introduciendo nuevos requisitos para su reconocimiento.

La primera de estas Comunidades Autónomas en desarrollar una normativa en este sentido fue Castilla León, con la entrada en vigor del Decreto 34/2016 de 22 de septiembre, sobre Entidades Asociativas Agroalimentarias Prioritarias y sus socios prioritarios de Castilla y León. En el Programa de Desarrollo Rural Castilla y León 2014-2020 se establece la preferencia de las mismas en la concesión de subvenciones y ayudas gestionadas por la Consejería de Agricultura y Ganadería. A diferencia de la normativa estatal, deja fuera del reconocimiento a las entidades civiles o mercantiles y establece un requisito de volumen de facturación único de veinte millones de euros con independencia del producto. Tampoco se exige la comercialización conjunta de la totalidad de la producción, sino que se establece una obligatoriedad de aportación a la EAPr de únicamente el $60 \%$ de la producción -un 50\% para los grupos cooperativos- o que el valor de las compras a la o a las EAPr sea igual o superior al $25 \%$ de las ventas de la explotación. Mientras que mantiene unas exigencias idénticas acerca del control democrático en su funcionamiento. Finalmente, introduce novedades como la obligatoriedad de que el número de socios sea superior a quinientos ${ }^{14}$, pudiéndose computar los socios de las empresas participadas por la cooperativa en más del $50 \%$; nuevos requisitos con respecto a los fondos propios y a los activos de la EAPr; y otros con un tratamiento más formal como los orientados a la profesionalización de la dirección de estas Entidades o como el tener el domicilio social y fiscal en Castilla y León y permanecer asociado al menos en la EAPr durante cinco años. En el periodo de un año desde que vio la luz dicho Decreto se ha conseguido que siete entidades obtengan dicho reconocimiento ${ }^{15}$.

La siguiente Comunidad Autónoma que desarrolló una normativa en la misma dirección fue Castilla- La Mancha con el Decreto 77/2016, de 13/12/2016, por el que se establecen los requisitos para el reconocimiento de entidades asociativas prioritarias de interés regional de Castilla-La Mancha (Eapir) y se crea su registro. En el Programa de Desarrollo Rural de Castilla la Mancha 2014-2020 se establece la preferencia de las mismas y de los productores agrarios que formen parte de estas EAPir, en las líneas de ayuda a favor de la competitividad, transformación, comercialización, promoción, internacionalización. En este caso se exceptúa del reconocimiento a los Grupos Cooperativos, debiendo tener el resto de entidades su domicilio social en Castilla-La Mancha y desarrollando con carácter principal su actividad en el territorio de la Comunidad Autónoma. Encontramos la misma exigencia de la comercialización conjunta de la totalidad del producto para el que solicita el reconocimiento, de la misma manera que existe una continuidad en cuanto a las previsiones relativas al control democrático en el funcionamiento de la EAPir. Las cuantías de volumen de facturación son inferiores a las de la normativa

14 Las cuantías de facturación y socios se reducirán a cinco millones de euros y ciento veinticinco socios, cuando el $80 \%$ de su producción se acoja a figuras de calidad legalmente establecidas, es decir, Denominaciones de Origen, Indicaciones Geográficas Protegidas, Marcas de Garantía, etc...

15 Datos obtenidos del Registro de entidades asociativas agroalimentarias prioritarias y de sus socios prioritarios de Castilla y León: AGROPAL, SOC. COOP.; SOCIEDAD COOP. GENERAL AGROPECUARIA ACOR; SOCIEDAD COOP. LTDA BAJO DUERO (COBADU); CONSORCIO DE PROMOCIÓN DEL OVINO S.C.; AVIGASE SCL.; COPISO SORIA SDAD COOP y ALTA MORAÑA, S. COOP. 
estatal, estableciendo para el reconocimiento de un producto determinado desde 25 millones de euros hasta los 45 millones (entre 20 y 650 en la normativa estatal) y para el reconocimiento genérico un volumen de facturación total anual superior a 100 millones de euros (750 millones en la normativa estatal). Hasta el momento no hay entidades constituidas como EAPir ${ }^{16}$.

Por último, la Comunidad Autónoma de Andalucía también ha llevado a cabo su regulación a través del Decreto 188/2017, de 21 de noviembre, por el que se regulan las entidades asociativas prioritarias agroalimentarias de Andalucía (EAPA) y se crea su Registro. Esta reciente normativa permite adquirir dicha calificación a las mismas entidades que la regulación estatal, siempre que más del 90\% de las personas productoras individuales que forman parte de la EAPA tengan la mayor parte de la superficie de sus explotaciones ubicadas en Andalucía y más del $90 \%$ de su actividad económica se desarrolle en dicha Comunidad. Al igual que la normativa estatal se introducen medidas para el control democrático de la entidad y se mantiene el requisito de la comercialización conjunta de la totalidad de la producción, salvo situaciones excepcionales (no determinadas en la norma) en las que se permite reducirla hasta el $85 \%$ del producto o productos para el que se solicita el reconocimiento. Finalmente, con respecto a los requisitos de facturación, mantiene unas cuantías inferiores a las estatales diferenciando según se solicite el reconocimiento de un producto determinado o el reconocimiento genérico, aunque para el genérico se observará el peso individual de cada una de las categorías de productos objeto de comercialización. Ahora bien, como novedad, introduce la posibilidad de adquirir la calificación a las entidades que no cumplen con las condiciones de facturación iniciales, exigiendo unas cuantías de facturación inferiores y un crecimiento acumulado de al menos un $20 \%$ en los tres últimos ejercicios previos a la solicitud. Si en este último caso no alcanzan el crecimiento exigido continuarán pudiendo adquirir la calificación siempre que se trate de una entidad resultante de una integración realizada en los seis meses anteriores a la solicitud.

\section{Conclusiones}

Aunque en un principio la Ley 13/2013 había generado muchísimas expectativas en el sector, todas estas dificultades y requisitos han pesado en las entidades a la hora de conseguir las condiciones exigidas, viéndose reflejado en el reducido número de EAP que han sido reconocidas durante los casi 4 años que lleva en vigor. Seguramente el requisito que más ha influido en ello ha sido el de facturación, las altas cuotas exigidas son casi inalcanzables por la mayoría de las entidades actuales, parece ser que la Ley está diseñada únicamente para las más grandes de cada sector. Quizás hubiera sido más conveniente la realización de un proyecto a largo plazo, en el que durante un periodo inicial se fomente la integración de las entidades con una facturación media, estableciendo unas cuantías inferiores a las fijadas en la actualidad, y que al cabo de los años se fuesen

16 Existen noticias de la intención de constituirse como EAPir de Oleotoledo, sin que estas intenciones se hayan materializado. 
progresivamente aumentando estas cuantías. Este planteamiento también ayudaría a disminuir el problema relacionado con la concentración/diferenciación, ya que sería más sencillo que se integren dos o tres entidades y alcancen unas cuotas inferiores, a que desde un principio se necesite la integración de 10 o 15 entidades para alcanzar estas cuotas superiores.

El papel de las Comunidades Autónomas en el fomento de la integración debe ser mayor, es necesario que continúen promoviendo el redimensionamiento ya que hasta el momento únicamente tres Comunidades han dado el paso a la hora de establecer una normativa. En este sentido, las normativas de Castilla-La Mancha y Andalucía continúan la misma línea fijada que la estatal, mientras que la normativa de Castilla y León se ha situado en una dirección diferente al no exigir la comercialización conjunta de totalidad del producto y no adaptar las exigencias de facturación a los de cada uno de los productos creando una discriminación entre entidades que se dedican a productos que generan una menor facturación.

Como se ha dicho, el éxito o fracaso de la ley puede valorarse en función de los resultados obtenidos, y en la actualidad únicamente se encuentran inscritas 6 EAP en el Registro Nacional de Entidades Asociativas Prioritarias: 1) EA GROUP S.C. que vino a sustituir a OVIESPAIN S.L, Cooperativa de Segundo Grado formada a su vez por otras dos cooperativas de segundo grado como son OVISO y CORDESUR, distribuida en las Comunidades Autónomas de Andalucía y Extremadura, obtuvo un reconocimiento específico para productos ovinos de leche y carne; 2) DCOOP S.C.A, Cooperativa de Segundo Grado integrada por 117 Cooperativas diferentes, distribuida en las comunidades de Andalucía Castilla-La Mancha y Extremadura, cuenta con un reconocimiento genérico ya que se dedica a varios sectores (Aceite, Aceituna de mesa, Vino, Ganadería, Cereales y Suministros); 3) AN S.C Cooperativa de Segundo Grado integrada por 118 Cooperativas, distribuida en las Comunidades de Aragón, Castilla - La Mancha, Castilla y León, Extremadura, La Rioja y Navarra, con un reconocimiento genérico (Cereales, Suministros y Servicio, Alimentación Animal, Frutas y Hortalizas, Avícola de Carne, Porcino Blanco); 4) INDULLEIDA S.A., Entidad Mercantil con más del 50\% del capital en manos de 7 Cooperativas de Primer y Segundo Grado, distribuida en las comunidades de Aragón, Cataluña, La Rioja y Navarra, cuenta con un reconocimiento específico en Transformados Hortofrutícolas; 5) IBERICO DE COMERCIALIZACION S.C.L, Cooperativa de Primer grado, distribuida en las Comunidades de Andalucía, Castilla y León y Extremadura, tiene un reconocimiento específico para Porcino Ibérico; 6) OVIARAGON, Cooperativa de Primer grado, distribuida en las Comunidades de Aragón, Andalucía, Baleares, C. Valenciana, Castilla la Mancha, Castilla y León, Cataluña, La Rioja, Navarra, tiene un reconocimiento específico para Ovino de leche y carne.

Habrá que prestar atención a cómo se siguen desarrollando las integraciones de estas entidades y si estas se siguen manteniendo en el tiempo, ya que el objetivo principal de la constitución como EAP debe ser el alcanzar una mayor dimensión para ganar en competitividad y no únicamente el recibir las ayudas como ocurre en algunos casos. 


\section{Referencias bibliográficas}

Berges Angós, I. (2014) Fusión de cooperativas y entidades asociativas agroalimentarias: Ley13/2013. Actualidad Jurídica Aranzadi, No 884, p. 7.

Calvo Vérgez, J. (2013) La nueva Ley 13/2013 y la creación de la figura de la 'entidad asociativa prioritaria': un paso adelante en la competitividad de las cooperativas agrarias. Actualidad Jurídica Aranzadi, № 871, p. 6.

Cano Ortega, C. (2015) Hacia la calificación como entidad asociativa prioritaria: las diferentes formas de integración de las cooperativas agroalimentarias. REVESCO. Revista de Estudios Cooperativos, Segundo Cuatrimestre, $\mathrm{N}^{\mathrm{o}} 118$, pp. 60-85. DOI: 10.5209/rev_REVE.2015.n118.49064.

Fayos Gardó, T. y Calderón García, H. (2013) Principales problemas de internacionalización de las cooperativas agroalimentarias españolas. REVESCO. Revista de Estudios Cooperativos, Segundo Cuatrimestre, $\mathrm{N}^{\mathrm{o}} 111$, pp. 32-59. DOI: 10.5209/rev_REVE.2013.v111.42675

Gallego Sevilla, L. (2011) Medios económicos y disciplinarios para combatir el incumplimiento de los socios de participar en las actividades de las cooperativas agrarias. REVESCO. Revista de Estudios Cooperativos, Primer Cuatrimestre, № 104 , pp. 63-101. DOI: 10.5209/rev_REVE.2011.v104.3.

Vargas Vasserot, C. (2010) Integración y diferenciación cooperativa: de las secciones a los grupos de sociedades. Boletín de la Asociación Internacional de Derecho Cooperativo = Journal International Association of Cooperative Law, № 44, pp. 159-176.

Vargas Vasserot, C. (2012) Las Sociedades Agrarias de Transformación. Empresas agroalimentarias entre la economía social y la del mercado. Madrid: Dykinson.

Vargas Vasserot, C. (2013) Impulso a la integración de cooperativas y otras empresas agroalimentarias a través de su posible calificación de Entidades Asociativas Prioritarias. Artículo publicado en Ccopyme.org, http://www.ccopyme.org/articulo.php?a=58. 